\title{
Subcellular protein extraction from human pancreatic
} cancer tissues

\author{
Anette Börner ${ }^{1}$, Uwe Warnken², Martina Schnölzer ${ }^{2}$, Jörg von Hagen ${ }^{3}$, Nathalia Giese ${ }^{4}$, Andrea Bauer ${ }^{1}$, and \\ Jörg D. Hoheisel ${ }^{1}$ \\ ${ }^{1}$ Functional Genome Analysis, Deutsches Krebsforschungszentrum, Heidelberg, Germany, 2 Protein Analysis, Deutsches \\ Krebsforschungszentrum, Heidelberg, Germany, ${ }^{3}$ Performance \& Life Science Chemicals, R\&D MDA Proteomics, Merck \\ $K G a A$, Darmstadt, Germany, and the 4 Department of General Surgery, University of Heidelberg, Heidelberg, Germany
}

BioTechniques 46:297-304 (April 2009) doi 10.2144/000113090

Keywords: cancer; disease; pancreas; protein extraction; protein fractionation

Proteins are the major class of effector molecules in cellular systems. For the identification of functional differences between normal and diseased tissues, a reliable analysis of their protein content is essential. Reproducible isolation and fractionation of intact proteins are important in this respect, but their complexity in structure and concentration, their close interaction, and their instability represent major challenges. For protein isolation in tissues, the breakdown of cell-cell and cell-matrix connections within a tissue without affecting protein quality is a critical factor. We compared different processes for a compartmental protein preparation from pancreatic tissue, one of the most challenging tissues for protein isolation because of its high protease content. Success of the different procedures varied greatly. Based on a scheme of tissue-slicing and subsequent cell isolation, we established a reliable workflow for the fractional extraction of cytosolic proteins, membrane and organelle proteins, nuclear proteins, and cytoskeletal filaments. The tissue slices also allow for a representative confirmation of individual samples' cellular status by histochemical processes, and a proper separation or mixing of cellular material from across a tumor if required.

\section{Introduction}

The challenges of studying the human proteome are numerous due to the huge complexity in size, structure and functionality of proteins; their diverse and frequent interactions and modifications; the enormous dynamic range in protein concentration; and the variation in the abundance at different locations within cells and tissues It is estimated that there are several hundred thousand to several million different human protein molecules (1), and only a minority of these are present in relatively large quantities (2). A reliable protein analysis has to be able to deal with these points sufficiently in order to record a snapshot of the proteomic status of cells or tissues.

A recent increased interest in analyzing the complete protein content of tissues has sparked fresh developments in the area of protein extraction. In order to reduce the complexity of the analysis, sample fractionation methods are being used. Besides the classical centrifugation-techniques $(3,4)$, chemophysical properties have also been utilized for separation (5). One of these methods is differential detergent fractionation (6), a sequential extraction process with detergent-containing buffers yielding up to four different subproteomic extracts, which are enriched in (i) cytosolic proteins, (ii) membrane and organelle proteins, (iii) nuclear proteins and (iv) cytoskeletal filaments. In addition to reducing fraction complexity, the molecules within each fraction also have more similar biophysical properties. Another advantage is the option to define the subcellular localization of proteins and thus monitor their compartmental redistribution at basal and stimulated conditions. Despite its advantages, differential detergent fractionation was established for cultured cells. Cultured cells are clearly artificial systems, and significant differences between the gene expression profiles of cell cultures and tissues have been reported (7). Additionally, as a consequence of the cell-cell and cell-matrix contacts in tissues, there are many differences in protein extractions between tissue and cultured cells. In tissue, the contacts have to be discon- nected and the cells isolated in a manner so that the detergent-containing buffer is able to reach the individual cells. Ideally, this should happen without affecting cell integrity. The nuclear membranes should not be disrupted during the process in order to avoid proteincompartment mixing prior to fractionation.

As part of a large-scale molecular analysis of pancreatic cancer [studying in each sample the methylation patterns of genomic DNA, transcript levels, and protein expression and modification (www.moldiagpaca.eu)], we compared different tissue preparation methods and established a workflow that allows protein extraction from pancreatic tissues. Pancreatic tumors are particularly difficult to handle due to the very high content of protein-degrading enzymes. Since working with various models, we aimed for a process that could be applicable to a number of human and other tissue sources. Initial analyses were therefore performed with pancreatic tissues from rat and pig in order to preserve less-abundant human samples. The established process yielded good-quality protein samples from both cell cultures 
A
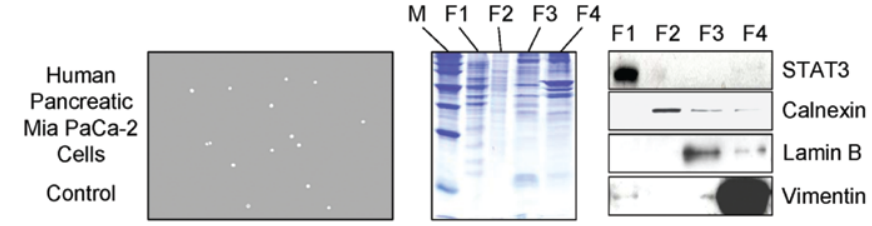

B
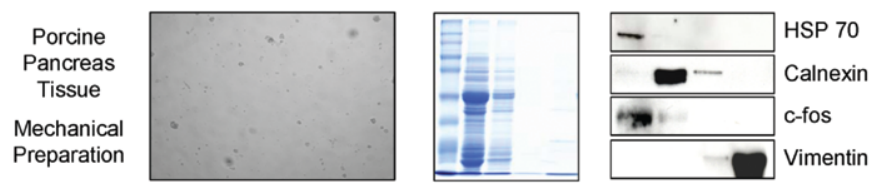

C
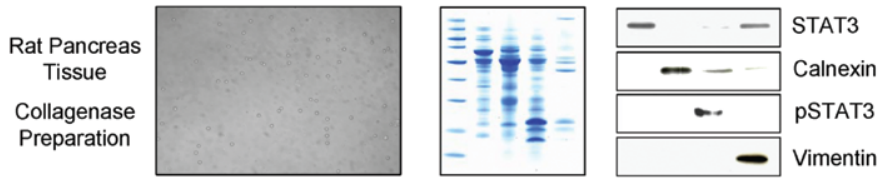

D

$$
\begin{aligned}
& \text { Human } \\
& \text { Pancreatic } \\
& \text { Tumour } \\
& \text { Tissues } \\
& \text { Collagenase }
\end{aligned}
$$
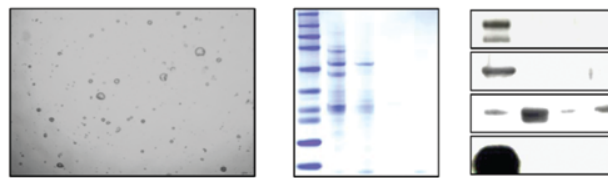

STAT3

Preparation

E
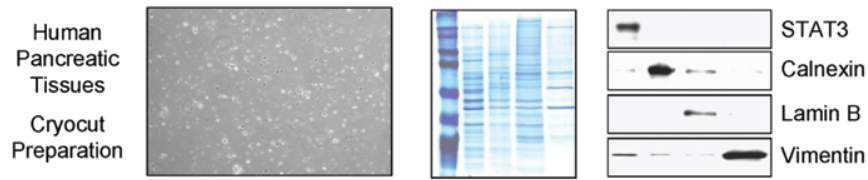

Figure 1. Comparison of the tissue preparation processes. For either tissue preparation method, a picture of the cells after the respective treatment is shown, next to a one-dimensional protein gel of the resulting fractions stained by Coomassie blue. Also, a Western blot analysis is presented, which indicates the distribution of the marker proteins used for the definition of the fractionation. The respective marker molecule is named at the right margin. The lanes of the gels and Western blots are labeled with M (marker), F1 (cytosolic fraction), F2 (membrane fraction), F3 (nuclear fraction) and F4 (cytoskeletal fraction).

and animal and human tissues, and the results were reproducible. In addition, the actual tissue samples could be checked for their tumor-cell content by histochemical analysis. Also, the process permits a split of each sample into three identical portions for a parallel analysis at the molecular level of DNA, RNA and protein.

\section{Material and methods}

Materials

All chemicals and solvents were purchased in extra-pure grade from Merck (Darmstadt, Germany) unless stated otherwise. Media and other solutions for cell culture were obtained from Invitrogen (Paisley, UK). Collagenase type XI was from Sigma (Taufkirchen, Germany).

Source of tissue samples

Fresh tissues from rat and pig were stored directly after resection in buffer containing protease inhibitors (Complete Mini Protease Inhibitor; Roche, Mannheim, Germany) and used for protein isolation the same day. For frozen porcine tissues, the samples were immediately snap-frozen in liquid nitrogen and stored at $-80^{\circ} \mathrm{C}$.

Human pancreatic specimens were collected during surgery on pancreatic cancer patients and samples were snapfrozen in liquid nitrogen directly after resection and subsequently stored at $-80^{\circ} \mathrm{C}$. Written informed consent was obtained from all patients. The study was approved by the local ethics committee of the University of Heidelberg.

Preparation of MIA PaCa-2 cells Cells of the pancreatic cancer cell-line MIA $\mathrm{PaCa}-2$ (8) were cultured in Dulbecco's modified Eagle medium (DMEM; Invitrogen) and $10 \%$ fetal calf serum at $37^{\circ} \mathrm{C}$ and $5 \% \mathrm{CO}_{2}$. The cells were detached from the flasks at $80 \%$ confluence by incubation in a solution of $0.05 \%$ trypsin, $0.53 \mathrm{mM}$ EDTA (Invitrogen) at $37^{\circ} \mathrm{C}$ for $5 \mathrm{~min}$. About $3 \times 10^{6}$ cells were used for each protein extraction.

Mechanical tissue preparation

Mechanical tissue preparation with mortar and pestle. Approximately $800 \mathrm{mg}$ of tissue sample were cut into small pieces with a scalpel and transferred into a mortar (Bürkle, Lörrach, Germany) filled with liquid nitrogen. The tissue was ground to a fine powder with a pestle in the continuous presence of liquid nitrogen and transferred into a reaction tube with Extraction Buffer 1 of the ProteoExtract Subcellular Proteome Extraction Kit (Merck) at $4{ }^{\circ} \mathrm{C}$.

Mechanical tissue preparation with a glass homogenizer. Two types of homogenizers were used: the DOUNCE S (VWR, Darmstadt, Germany) with a clearance of 10-30 um between pestle and tube and the DOUNCE L (VWR) with a clearance of 50-70 $\mu \mathrm{m}$. Approximately $800 \mathrm{mg}$ of frozen or fresh tissue were added to $3 \mathrm{~mL}$ of Extraction Buffer 1 of the ProteoExtract Subcellular Proteome Extraction Kit at $4^{\circ} \mathrm{C}$ and homogenized on ice for $20 \mathrm{~s}$.

Collagenase digest

Approximately $800 \mathrm{mg}$ of fresh tissue samples were cut into small pieces with a scalpel. The tissue pieces were incubated in a solution of collagenase type XI. Collagenase with an activity of $1380 \mathrm{U} / \mathrm{mg}$ was dissolved to a final concentration of $1 \mathrm{mg} / \mathrm{mL}$ in Hank's buffer $(137 \mathrm{mM} \mathrm{NaCl}, 5.4 \mathrm{mM}$ $\mathrm{KCl}, 0.8 \mathrm{mM} \mathrm{MgSO}_{4}, 0.3 \mathrm{mM} \mathrm{Na}_{2} \mathrm{HPO}_{4}$, $0.4 \mathrm{mM} \mathrm{NaH}_{2} \mathrm{PO}_{4}, 1.3 \mathrm{mM} \mathrm{CaCl}_{2}, 4.2$ $\mathrm{mMNaHCO}_{3}$, and $10 \mathrm{mM}$ HEPES supplemented with $0.4 \%$ BSA). Twelve milliliters of Hank's buffer (16,560 U of collagenase) were used for $400 \mathrm{mg}$ tissue. The sample was incubated at $37^{\circ} \mathrm{C}$ for $16 \mathrm{~min}$. In order to stop the enzyme reaction, $10 \mathrm{~mL}$ of cold $\left(4^{\circ} \mathrm{C}\right)$ Hank's buffer were added. After the cells had sedimented, the supernatant was removed and the cells were transferred into centrifuge tubes, in which they were centrifuged at $300 \times g$ at $4^{\circ} \mathrm{C}$ for $3 \mathrm{~min}$. The supernatant was removed. The pellet was taken up in cold Hank's buffer, centrifuged a second time and the cells were again taken up in 3 $\mathrm{mL}$ Hank's buffer.

Preparation of frozen human pancreas tissue samples by cryocut section

Without thawing, each tissue was cut into slices of 20- $\mu \mathrm{m}$ thickness with a cryotome (Leica CM 1850 UV; Leica, Wetzlar, Germany) at $-34^{\circ} \mathrm{C}$. As a control of tumor status, several $10-\mu \mathrm{m}$ slices were prepared in between and used for histological staining. To ensure an even distribution of the different areas of a tumor, the slices of each sample were mixed prior to being split for the separate protein, DNA and RNA isolations. For protein extraction, the tissue slices were covered with liquid nitrogen and gently ground by three turns with a polypropylene micropestle (Eppendorf, Hamburg, Germany) that fit into 2-mL Eppendorf 
Table 1. Typical Proteins Identified by Mass Spectrometry in the Four Subcellular Extracts
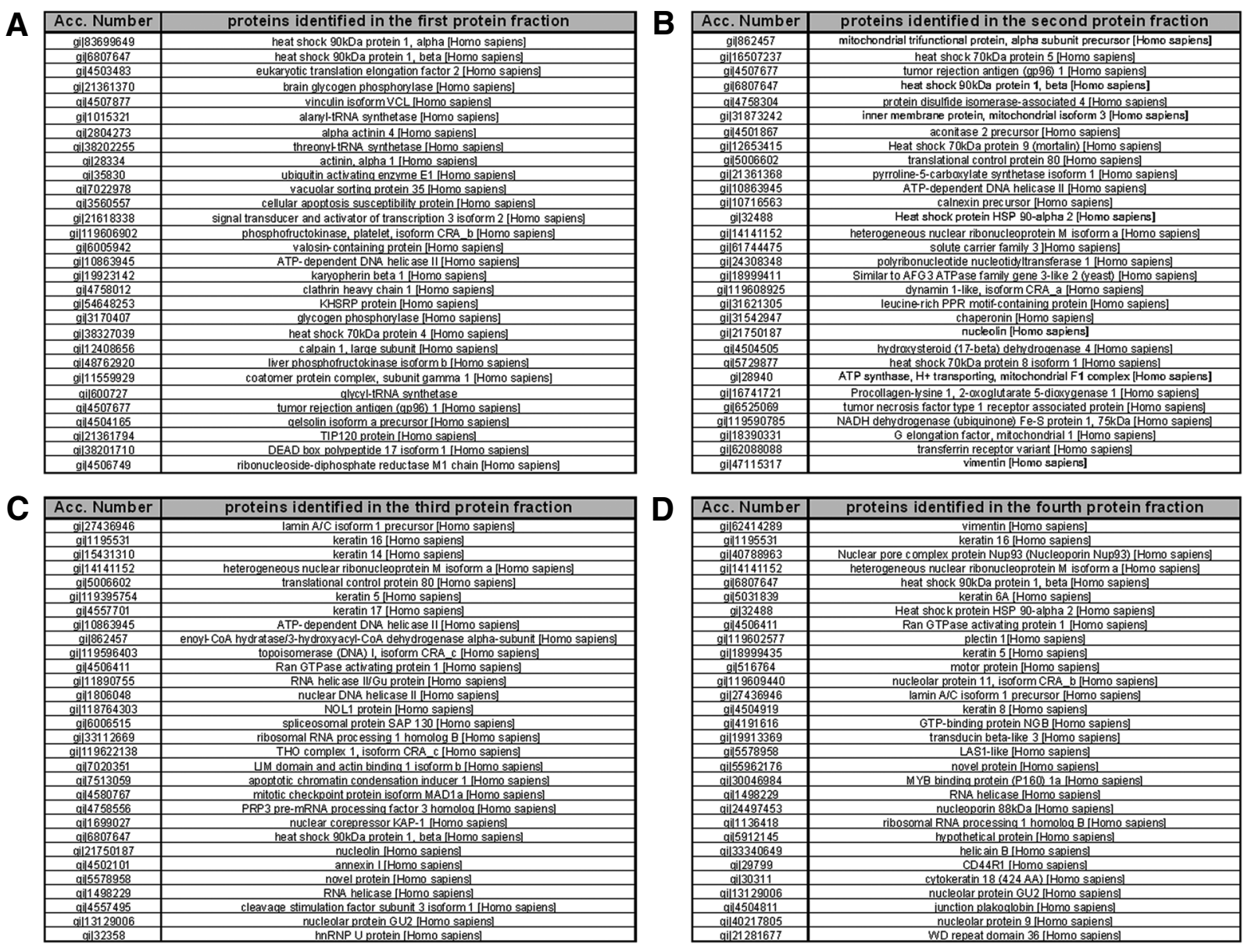

The 30 most abundant proteins of each subcellular fraction are listed. (A) Cytosolic; (B) membranous; (C) nuclear; (D) cytoskeletal.

tubes. About $20 \mathrm{mg}$ of the tissue powder were used for $1 \mathrm{~mL}$ Extraction Buffer 1 of the ProteoExtract Subcellular Proteome Extraction Kit.

Control of cell integrity

In order to determine the status of the cells after the initial preparative steps, the loss of membrane integrity indicated by the inability of the cells to exclude Trypan blue was used to measure the cell status using a hemocytometer (9). The cell-suspension resulting from a tissue preparation was incubated with an equal amount of $0.1 \%$ Trypan blue stain (Greiner Bio-One, Frickenhausen, Germany) and studied under a microscope.

Protein extraction and fractioning For protein extraction, the ProteoExtract Subcellular Proteome Extraction Kit was used according to the manufacturer's recommendations unless stated otherwise. By this procedure, based on the work of Ramsby and Makowski (6), proteins are divided into four fractions containing the cytosolic proteins, membrane and organelle proteins, nuclear proteins and cytoskeletal filaments, respectively. In short, Extraction Buffer 1 was added to the cells or tissue powder. After a centrifugation at $1000 \times g$ for $10 \mathrm{~min}$, the supernatant was removed as cytosolic fraction. The pellet was resuspended in Extraction Buffer 2, which solubilized membrane proteins. After another centrifugation step at $6000 \times g$ for $10 \mathrm{~min}$ and collecting the supernatant as membranous fraction, Extraction Buffer 3 was used for the solubilization of nuclear proteins. Finally, the cytoskeletal proteins were pelleted at $6800 \times \mathrm{g}$ for $10 \mathrm{~min}$ and resuspended in Extraction Buffer 4. The protein concentration was determined with the DC-Protein Assay (Bio-Rad, Munich, Germany) in microtiter plate format.

One-dimensional polyacrylamide gels Proteins were separated on $10 \%$ polyacrylamide gels. Approximately two to five micrograms of protein were loaded per lane. Rather than equal amounts, equal volumes were loaded to maintain the existing proportion of the proteins in the different extracts. Electrophoresis was performed using a Mini-PROTEAN 3 Electrophoresis Cell System (Bio-Rad) with stacking gels of $5 \%$ and separating gels of $12 \%$ polyacrylamide in $25 \mathrm{mM}$ Tris- $\mathrm{HCl} \mathrm{pH}$ 8.3, $0.18 \mathrm{M}$ glycine and $0.1 \% \mathrm{SDS}$. Perfect Protein Marker (range 10-225 kDa) (VWR, Darmstadt, Germany) or full-range Rainbow molecular weight marker (GE Healthcare, Munich, Germany) were used as molecular mass markers. After electrophoresis, protein bands were detected by Coomassie Brilliant Blue G250 staining (Merck) or immunoblot analysis.

Immunological validation of protein extraction After electrophoresis, the proteins were blotted to a polyvinylidene fluoride (PVDF) Western blot membrane $(0.45 \mu \mathrm{m}$; Roche, Mannheim Germany) at $0.8 \mathrm{~mA}$ / $\mathrm{cm}^{2}$ for $1 \mathrm{~h}$. Subsequently, the membrane was blocked with 3\% TopBlock (Fluka, Deisendorf, Germany) at room temperature for $4 \mathrm{~h}$. Incubation with primary 


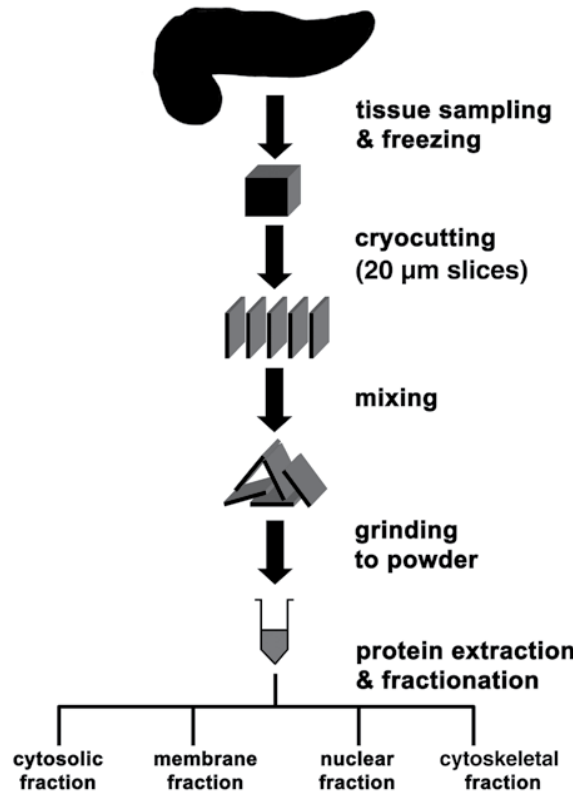

Figure 2. Workflow of the subcellular protein extraction process. Directly after sampling, pancreas tissue was snap-frozen in liquid nitrogen. Each tissue was cut into slices of 20- $\mu \mathrm{m}$ thickness. Prior to subcellular protein extraction, the slices were mixed and ground to powder.

antibody was performed in 3\% TopBlock in PBS-T $(0.1 \%$ Tween-20 in phosphate buffered saline (PBS), $10 \mathrm{mM} \mathrm{Na}_{2} \mathrm{HPO}_{4}$, $1.75 \mathrm{mM} \mathrm{KH}_{2} \mathrm{PO}_{4}, 13.7 \mathrm{mM} \mathrm{NaCl}$ and $2.68 \mathrm{mM} \mathrm{KCl}$ ) overnight. The following primary antibodies were used: antiCalnexin (1:2000 dilution), anti-lamin B (1:500), anti-HSP70 (1:1000) (Calbiochem, Schwalbach, Germany), anti-c-fos (1:1000), anti-Vimentin (1:2000) (Sigma), anti-STAT3 (1:1000) and anti-phosphoSTAT3 (1:500) (Cell Signaling, Danvers, USA). Subsequently, incubation with a horseradish peroxidase (HRP)-labeled secondary antibody was performed for detection. Anti-rabbit antibody was obtained from Sigma (Cat. no. A 6159) and anti-mouse antibody was from Acris (Cat. no. R1349HRP; Hiddenhausen, Germany). Incubation was done in 3\% TopBlock in PBS-T at room temperature for $4 \mathrm{~h}$. Chemiluminescence was detected by the ECL Western blotting detection reagent and Hyperfilm (GE Healthcare) as recommended by the manufacturer.

\section{Two-dimensional PAGE}

Separation was performed with the Ettan DALT system (GE Healthcare). The protein samples were applied onto Immobiline Dry Strips pH 3.0-10.0 NL strips (GE Healthcare). From each sample, $100 \mu \mathrm{g}$ of protein were loaded. For later estimation of the molecular weight, a molecular standard mixture (Sigma) was added. The two-step equilibration after isoelectric focusing was
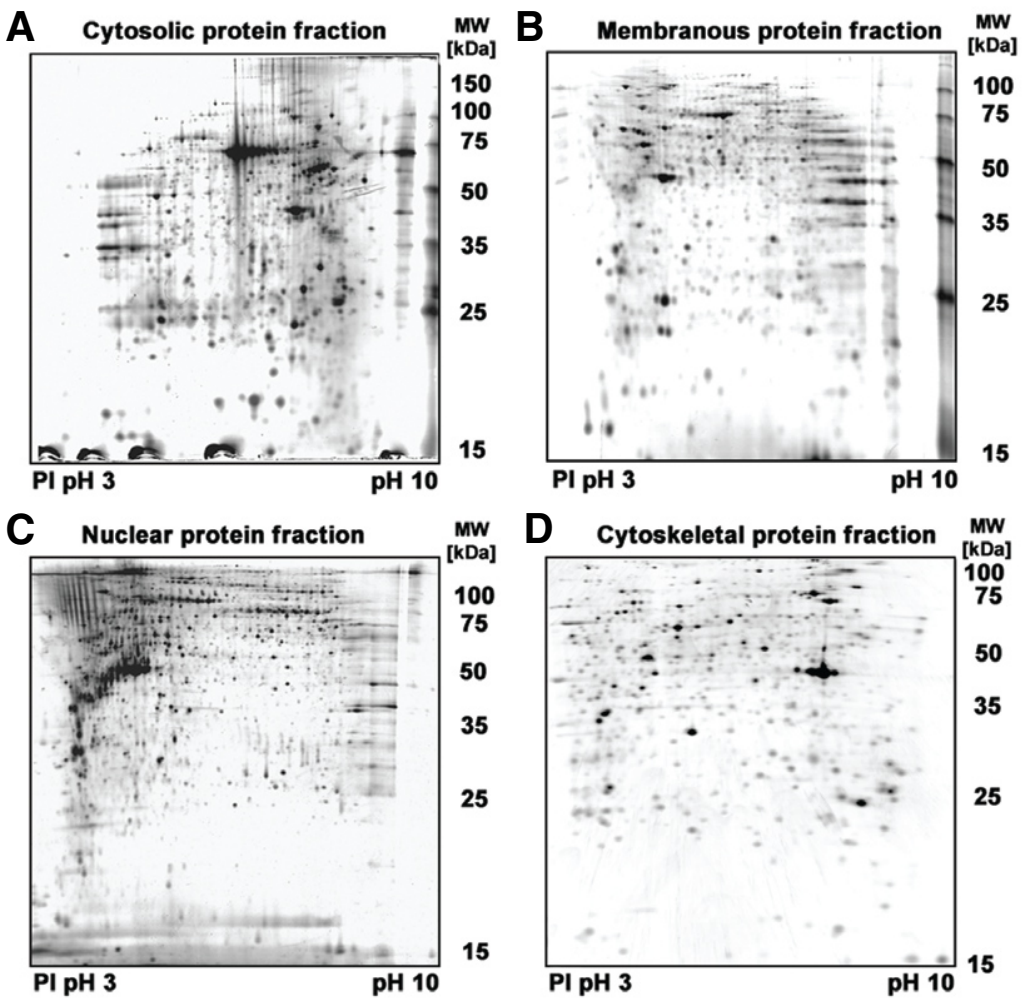

Figure 3. Silver-stained two-dimensional gels of protein fractions. Typical results of cytosolic (A), membranous $(B)$, nuclear $(C)$, and cytoskeletal (D) protein extracts are shown, which were isolated by the cryocut section process from cancerous human pancreas tissue.

performed with $10 \mathrm{mg} / \mathrm{mL}$ dithiothreitol shaking for $30 \mathrm{~min}$ and $25 \mathrm{mg} / \mathrm{mL}$ iodoacetamide shaking for another $30 \mathrm{~min}$. The second dimension electrophoresis was done on $12 \%$ polyacrylamide gels in $25 \mathrm{mM}$ Tris-HCl ( $\mathrm{pH}$ 8.3), $0.18 \mathrm{M}$ glycine and $0.1 \%$ SDS at $1.5 \mathrm{~W}$ for $5-7 \mathrm{~h}$. To visualize the protein spots in the gel, the silver staining procedure described by Sinha et al. (10) was employed, which is compatible with protein identification by mass spectrometry.

\section{Protein spot detection}

The silver-stained two-dimensional gels were scanned in 16-bit mode (300 dpi) using a UMAX Image Reader (GE Healthcare), saved as a gray-scale TIFF file, and analyzed by the image analysis software Proteomweaver, version 3.1 (Bio-Rad). Parameters of intensity, contrast and radius were chosen so that $800-1000$ protein spots per gel were calculated. All other parameters were set as proposed by the manufacturer.

\section{In-gel tryptic digestion}

Gel bands of interest were excised and digested in-gel with trypsin (Promega, Madison, USA) overnight. For MALDIMS analysis, the supernatant from the tryptic digestion was used directly, whereas for ESI-MS/MS analysis, peptides were extracted from the gel bands. Extracts from steps 1 [acetonitrile $(\mathrm{MeCN}) / \mathrm{H}_{2} \mathrm{O}$ / formic acid (FA), 50.0\%/49.9\%/0.1\%, $\mathrm{v} / \mathrm{v} / \mathrm{v}], 2(100 \% \mathrm{MeCN}), 3\left(\mathrm{H}_{2} \mathrm{O} / \mathrm{FA}\right.$, $99.9 \% / 0.1 \% \mathrm{v} / \mathrm{v})$ and $4\left(\mathrm{MeCN} / \mathrm{H}_{2} \mathrm{O} / \mathrm{FA}\right.$, $50.0 \% / 49.9 \% / 0.1 \% \mathrm{v} / \mathrm{v} / \mathrm{v}$ ) were combined, evaporated to dryness, and dissolved in $\mathrm{H}_{2} \mathrm{O} / \mathrm{FA}, 99.9 \% / 0.1 \%$, v/v.

Mass spectrometry

NanoLC-ESI-MS/MS analysis was performed using the CapLC capillary LC system (Waters, Eschborn, Germany) coupled to a hybrid quadrupole orthogonal acceleration time-of-flight tandem mass spectrometer (Q-TOF; Micromass/Waters, Manchester, UK) using a short HPLC gradient and parameter settings as described (11). Processed data were searched against the NCBInr database using the Mascot algorithm version 2.1.0 (MatrixScience Ltd., London, UK). The following search parameters were selected: taxonomy human; fixed carbamidomethyl modification on cysteine side chain; variable modification due to methionine oxidation; one missed cleavage site in the case of incomplete trypsin hydrolysis. The mass tolerance was set to $200 \mathrm{ppm}$ for precursor ions and $0.1 \mathrm{Da}$ for fragment ions. No fragment ion score cutoff was applied when performing the search. Any protein identified by only one peptide or that lacked at least two peptides found to 


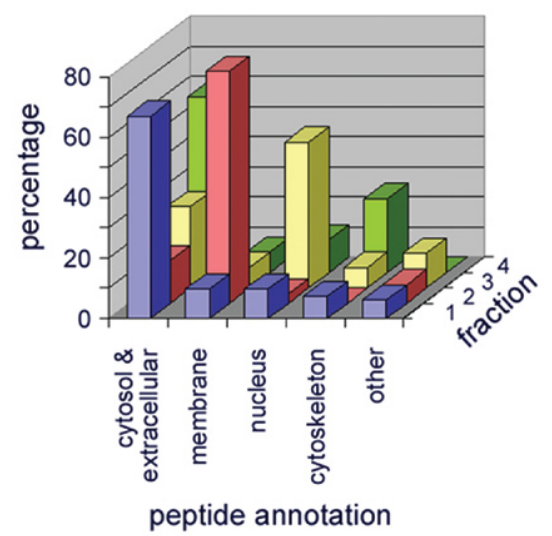

Figure 4. Distribution of proteins in the subcellular fractions as identified by mass spectrometry. Fractions 1-4 correspond to the cytosolic, membrane, nuclear, and cytoskeleton fraction, respectively. Assignment to the cellular locations was performed according to the Gene Ontology (GO) terms associated with the respective proteins.

have a significant identification score was ignored. Also, proteins identified with a Mascot score $<50$ were also not taken into account.

\section{Results}

Definition of marker proteins

As a quality control for the fractionation of the total proteome into four portions, marker-proteins were selected, which are known to be specific for one of the four fractions. For the cytosolic fraction, the proteins HSP70 and STAT3 were chosen. HSP70 (heat-shock protein 70) is a cytosolic protein, which supports the folding of other proteins. As a response to stress, HSP70 is overexpressed (12). The protein STAT3 (signal-transducer and activator of transcription 3) plays an important role in cytokine signal transduction. It is a cytosolic protein which can be activated by adding a phosphorous group. By phosphorylation, STAT3 is transformed to phospho-STAT3 and translocates to the nucleus, where it functions as a transcription factor (13). Therefore, the phosphoSTAT3 protein was selected as a nuclear protein marker. Calnexin was chosen as a marker-protein for the membrane-protein fraction, because it is an integral membrane protein of the endoplasmatic reticulum. It associates transiently with many different newly synthesized proteins to support their folding (14). Besides phospho-STAT3, the proteins c-fos and lamin B were selected as marker proteins for the nuclear fraction. C-fos is a nuclear phosphoprotein, which forms a tight but non-covalently linked complex with the JUN/AP-1 transcription factor (15). This complex interacts with DNA. Lamin B is an element of the nuclear lamina, a fibrous layer on the nucleoplasmic side of the inner nuclear membrane (16). As a marker of the cytoskeleton fraction, the protein vimentin was chosen. It is a class-III intermediate filament found in various non-epithelial cells (17).

\section{Protein extraction from}

MIA PaCa-2 cells

Initially, protein extraction was performed on cultured cells of the pancreatic cancer cell line Mia PaCa-2 as a control of the performance of the extraction and fractionation process. After detaching the cells from the surface of the culture flasks with a trypsin/EDTA solution, the cell integrity was checked by microscopy after Trypan blue staining. The cells were intact and no impurities could be seen (Figure 1A). Protein isolation and fractionation into cytosolic, membranous, nuclear and filamentous extracts was performed with the ProteoExtract Subcellular Proteome Extraction Kit. In an extraction from about $3 \times 10^{6}$ cells, approximately $600 \mu \mathrm{g}$ of protein were detected in the cytosolic fraction, $280 \mu \mathrm{g}$ in the membranous fraction, $300 \mu \mathrm{g}$ in the nuclear fraction, and $200 \mu \mathrm{g}$ in the cytoskeleton fraction. The four extracts were analyzed by one-dimensional SDS-PAGE and Coomassie blue staining. In the analysis, patterns were obtained that are typical of the four fractions (Figure 1A). Little to no degradation of the proteins was observed. To validate the separation into compartmental fractions, immunoblot analyses were performed. As expected, the marker molecules were detected in the appropriate fractions. These results formed the basis for a comparison of the various tissue preparation techniques.

\section{Mechanical tissue preparation}

The principle of mechanical cell isolation is the destruction by mechanical force of the cell-cell and cell-matrix connections within a tissue. For an initial analysis, we prepared porcine pancreas by grinding the tissue to a powder in liquid nitrogen using a mortar and pestle. The tissue samples stayed completely frozen during the entire process, inhibiting any protease activity. The tissue powder has the advantage of a good surfaceto-volume ratio, which permits the proteins' rapid contact with extraction buffers (18). However, visual examination by microscope of the cell status after grinding showed that the cells were completely destroyed by this treatment (data not shown). No organelles, like the nucleus, nor any bigger membrane parts could be seen, possibly due to shearing forces being too strong and causing destruction of cellular compartments.

Another, milder technique utilizes a glass Potter-Elvehjem homogenizing device

\section{E $=$ Introducing NBS Galaxy ${ }^{\circledR}$ $\mathrm{CO}_{2}$ Incubators. \\ The Cell Culture Problem Solvers.}

Problem 4: When we place cultures on the top shelf of our current incubator, they desiccate and grow differently then identical cultures on the lower shelves.

Solution: NBS Galaxy Incubators solve that problem by using a fanless, gentle convection to bathe the cells. This results in superior uniformity and an environment conducive to uniform cell growth with minimal desiccation.

Problem 5: Our lab wants to begin experimenting with hypoxic conditions and we're looking for an entry level incubator.

Solution: NBS' Galaxy 48R and 14 S are two models ideal for beginning hypoxic research. The convenient 48L/1.7 cu. ft. and $14 \mathrm{~L} / 0.5 \mathrm{cu}$. ft. sizes not only easily fit on the lab bench but also significantly reduce costly gas consumption seen in larger volume units.

Problem 6: Our $\mathrm{CO}_{2}$ incubator requires frequent and costly internal HEPA filter changes to maintain a sterile environment. In addition, it needs to be removed and replaced during any sterilization process, reintroducing contaminants into the disinfected unit.

Solution: NBS $\mathrm{CO}_{2}$ incubators have no internal HEPA filter, and therefore eliminate this recurring operating cost.

New Brunswick Scientific - A culture of innovation.

\section{New Brunswick}

www.nbsc.com/BT8

877-723-3318・bioinfo@nbsc.com 
$(19,20)$. It consists of a glass tube and a pestle whose width very nearly equals the tube's inner diameter (low clearance). Squeezing tissue through the clearance between tube and pestle disrupts intercellular connections (the smaller the gap, the higher the shearing force). Again, however, a control of the cell status after homogenization showed that the cells were damaged too much by the process, which tried clearances of 10-30 $\mu \mathrm{m}$ and $50-70 \mu \mathrm{m}$. The plasma membranes and membranes of the organelles-including the nuclear membranes-were destroyed. Gel-based analysis confirmed the disintegration of the cellular compartments (Figure 1B). In an extraction from $175 \mathrm{mg}$ pancreas tissue, approximately $3500 \mu \mathrm{g}$ and $2130 \mu \mathrm{g}$ of protein were detected in the cytosolic and the membranous fraction, respectively, with only $115 \mu \mathrm{g}$ present in the nuclear fraction and $285 \mu \mathrm{g}$ in the cytoskeleton fraction. Since the subcellular extraction method takes advantage of different protein solubility, complete cellular integrity is not required. As could be seen in immunoblot analyses, some minor fractionation did occur. However, the nuclear protein c-fos, for example, was identified in the first fraction, in which only cytosolic proteins were expected, clearly documenting the early disintegration of the nucleus.

\section{Collagenase digest}

Enzymatic digestion of pancreas tissue is often accomplished with collagenase. Commonly, this technique is described for the transplantation of islet cells or insulin studies (21-23) since for these applications, the isolated islet cells still have to fulfill their function after digestion. This fact suggested an interesting approach to our studies. For test purposes, $1.7 \mathrm{~g}$ fresh rat pancreas tissue, in small pieces, were initially incubated in collagenase solution. As visualized by microscopy, the cells were isolated with an intact plasma membrane, and the subsequent subcellular protein extraction was successful (Figure 1C). Approximately $2770 \mu \mathrm{g}$ of protein were detected in the cytosolic fraction, $3890 \mu \mathrm{g}$ in the membranous fraction, $1490 \mu \mathrm{g}$ in the nuclear fraction, and $725 \mu \mathrm{g}$ in the cytoskeleton fraction. Tests with frozen pancreas tissues failed since digestion occurred very unevenly. While some portions of tissue were not digested properly, cells and cellular organelles were already destroyed in other areas of the sample.

Worse, in contrast to the good results obtained with fresh rat tissues, the digest did not perform sufficiently reproducible with fresh human pancreatic tumor samples. Although all samples were treated in exactly the same way (in part by using identical buffers and solutions in parallel), the results varied substantially. While fractionation was successful in about one third of the cases, the proteome could not be split into the four subcellular extracts during the fractionation process on another third. In the example shown in Figure 1D, the extraction from $783 \mathrm{mg}$ frozen pancreas tissue produced $50 \mu \mathrm{g}$ of protein in the cytosolic fraction, $80 \mu \mathrm{g}$ in the membranous fraction, $50 \mu \mathrm{g}$ in the nuclear fraction, and $150 \mu \mathrm{g}$ in the cytoskeleton fraction. For the final, remaining third of the frozen tissue samples, the yield of protein was too low to be analyzed at all. The biggest part of cell protein got lost during the cell sedimentation step after digestion (see the Materials and methods section), since the cells had already been destroyed during digestion. The results did not appear to correlate with tumor grade or other factors. Mollenhauer et al. (24) and Imamura et al. (25) have reported significant differences in the amount of collagen and collagen subtypes present in human cancerous pancreatic tissue versus normal pancreatic tissue, and also strong variations among different tumors. These differences could very well be the reason for the unpredictable nature of the enzymatic preparation process.

\section{Preparation of human pancreas} tissue samples by cryocut section Another means of tissue preparation is the cryocut section system. As an alternative to sections made from formalin-embedded tissue, cryocut sections are commonly used for immunohistochemistry, as well as studies on pancreatic cancer. Both proteins and nucleic acids can be examined this way $(26,27)$. Snap-frozen pancreatic tissues were cut into $20-\mu \mathrm{m}$ slices. The diameter of a typical eukaryotic cell is $10-100 \mu \mathrm{m}$, and the diameter of the nucleus is about $5 \mu \mathrm{m}$ (28). Though many of the cells are cut during this process, the probability of destroying the nuclei is lower. The slices were transferred into 2-mL Eppendorf tubes filled with liquid nitrogen and gently ground to a powder with a micropestle that fit the shape of the reaction tube. (For an overview of this procedure, a workflow is shown in Figure 2.) During the entire process, the tissue was kept frozen to avoid protein degradation and thawing occurred only upon the addition of the first extraction buffer, which contained protease inhibitors. As mentioned before, tissue powder permits the rapid contact of cells and organelles to the extraction buffer, so proteases were blocked throughout the process (which is necessary when working with enzyme-rich pancreas tissue). A microscopic analysis of the resulting cell suspension showed individual cells, bigger membrane pieces, and intact nuclei (Figure 1E). Although the integrity of many of the cells was destroyed during the process, the compartments of the cells may have still existed. In contrast to the destruction observed in grinding whole tissues in liquid nitrogen with mortar and pestle, much weaker mechanical forces were needed on the cryocut slices, which in turn reduced substantially the damage done to the cells. As a result, the total proteome of frozen human pancreas tissue could be divided into cytosolic, membranous, nuclear, and cytoskeletal protein fractions according to the protein patterns and distribution of marker molecules. Results of similar quality were also obtained from rat and porcine tissues. A typical protein extraction from $50 \mathrm{mg}$ tissue powder yielded about $300 \mu \mathrm{g}$ protein in the cytosolic fraction, $100 \mu \mathrm{g}$ in the membranous fraction, $120 \mu \mathrm{g}$ protein in the nuclear fraction, and $90 \mu \mathrm{g}$ in the cytoskeletal fraction.

Another advantage of this method is the option to control each particular tissue sample for its tumor status. Individual tissue slices were transferred to glass slides, stained via appropriate histological procedures, and studied by pathologists for a precise classification. This control step is very helpful, since pancreatic cancer samples can be very inhomogeneous. In addition, since nearby tissues must be removed in addition to genuine tumor tissue to avoid cancer relapse, pancreatic cancer resections are often a mixture of tissue types. For a comparison of different tumor specimens where only tumor cells should be considered, it is crucial to avoid samples with a high content of necrotic, fatty, connective, or inflammatory tissue, which thereby increases the accuracy and reproducibility of an analysis.

By cutting a tumor sample into many thin slices, individual areas of a tumor could be analyzed in order to see differences between regions. In our analysis, the slices are utilized to the opposite effect. We usually simultaneously analyze the genomic DNA, RNA, and the protein content of tumors. Although methods exist that allow the extraction of these molecule classes from a single piece of tissue, we prefer to perform them separately, and we mix the tissue slices prior to splitting them for the three isolation procedures. This ensures an equal representation of the entire tumor in each analysis type. To visualize the quality of the protein fractionation from human pancreatic cancer tissue, gel electrophoresis and subsequent silver staining was performed (Figure 3).

\section{Gene Ontology analysis of fractions} protein content

While analysis by one-dimensional gel electrophoresis and the identification of marker proteins was sufficient for evaluating the basic processes, a thorough 
assessment of the cryocut procedure's performance required the identification of proteins in each of the extracted fractions. To verify that the proteins' presence in the respective extracts corresponded with their cellular localization, proteins from each extract were identified and reviewed with the help of Gene Ontology (GO; www. geneontology.org) terms. In order to achieve comparability between the four fractions, they were subjected to one-dimensional gel electrophoresis and for each fraction, a Coomassie-stained gel band was cut at the molecular weight of $80 \mathrm{kDa}$. The proteins in the gel pieces were digested with trypsin and subjected to a peptide identification by NanoLC-ESI-MS/MS analysis.

In total, 642 proteins were analyzed and 568 were identified by Ingenuity Pathway Analysis software (version 7.1; Ingenuity Systems, Redwood City, CA, USA), which was used to allocate the GO annotations. Sixty-one percent of the proteins in the cytosolic fraction were assigned by their $\mathrm{GO}$ terms to be present in the cytosol or extracellular space. For the membrane and nuclear fractions, $77 \%$ and $48 \%$ were assigned to the appropriate cellular location (Figure 4 ), respectively. Only in the cytoskeletal fraction were more proteins found that belonged to the cytosol and extracellular space $(58 \%)$ rather than being cytoskeletal filaments $(24 \%)$. This result is not surprising, however, since the fourth extraction buffer contains sodium dodecyl sulfate (SDS). SDS denatures proteins and thus solubilizes even proteins with low solubility: Besides the poorly-soluble cytoskeleton, proteins of the extracellular matrix could also be found in this fraction. In Table 1 , the 30 most abundant proteins of each fraction are listed (note that proteins that were not named by the software are shown as "others").

\section{Discussion}

By comparing different procedures of breaking up the cell-cell and cell-matrix connections in a tissue prior to differential detergent fractionation, we established a process that allows the protein extraction and fractionation in a highly reproducible manner, even from protease-rich pancreatic cancer tissues. Pancreas tissue was used since its analysis is of particular interest to us in an ongoing large-scale study (www. moldiagpaca.eu). However, because of its high level of proteases, it is also one of the most challenging tissues with respect to protein isolation. The advantage lies in the fact that if a method is successful with pancreatic cancer tissue, it is likely to be applicable to other tissues as well.

For a subcellular protein extraction, it is not obligatory that the cells' integrity stay intact completely. However, cellular compartments should not be destroyed during the process. Mechanical tissue preparation methods by mortar or homogenizer destroyed nuclei and other organelles. The use of collagenase digestion was hindered by significant differences in the amount of collagen that is present in human pancreatic samples $(24,25)$. Determining the collagen amount in each specimen was impractical, while completing digestion by an elongation of the incubation time or an increase of the collagenase concentration, on the other hand, created a problem with the protein integrity due to the tryptic activity of colla- genase. Addition of a large amount of collagenase obscured subsequent analyses.

The cryocut process provided a reliable solution. The process works only with frozen tissue, though from a practical standpoint this is the most relevant kind of sample in a clinical setting; fresh tissue is also easily frozen. Cryocut slices enable the confirmation of cellular status by histochemistry. and proper mixing of cellular material from across a tumor can be achieved if required. The slices are ground in liquid nitrogen with much less mechanical force than that required for whole tissues. While the treatment disrupts some cells, the cell

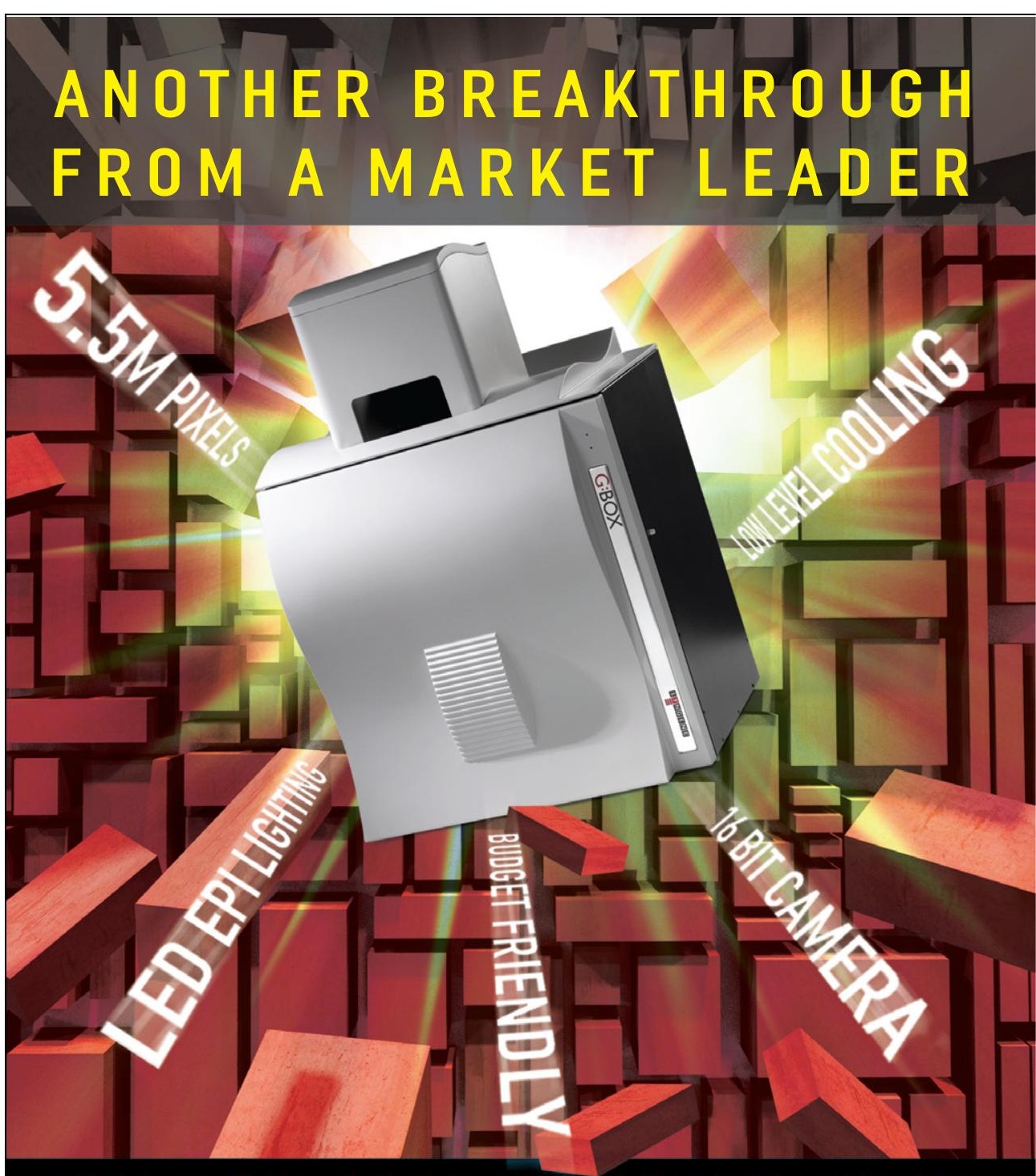

THE G:BOX EF FLUORESCENCE IMAGING SYSTEM

Our best yet! Not only do we have a cooled, true 16 bit camera for the ultimate dynamic range, but we also have mega pixel resolution, extended exposure times and LED lighting all for the price of a basic system. You have to see it to believe the punch we've packed into this package! 
organelles stay intact, which permits appropriate fractionation. By performing the grinding step, the individual cells are easily subject to detergents and protease inhibitors upon addition of the initial buffer, markedly improving protein stability compared to incubating the slices directly.

The investigation of sub-proteomes offers an advantage over analyzing total cell lysates, since sample complexity is reduced. Furthermore, it allows the investigation of translocation effects of treated/ untreated or cancer/non-cancer tissues, for example. Also, the resulting protein extracts share some biophysical parameters, which simplify subsequent analyses. However, it should be noted that the subdivision process is only an enrichment based on differences in solubility. Moreover, proteins may be present in more than one cellular compartment. One example for this is annexin A2: Monomeric annexin A2 is mainly located in the cytosol; elevation of intracellular calcium induces translocation to the cytoskeleton; a heterodimer composed of annexin A2 and 3-phosphoglycerate-kinase is located in the nucleus; and the heterotetrameric form, composed of two annexin A2 monomers and a p11 dimer, is primarily found at the plasma membrane (29).

The process described here forms the basis for a currently, broader study based on the analysis of a large number of pancreatic tissue samples. Therein, tissue protein data are immediately interrelated to variations at the RNA and epigenetic levels of the corresponding genes in the same tissues, and compared alongside protein analyses of the patients' sera and pancreatic excretions. We hope that this work will lead to significantly improved diagnostic means for earlier detection of the disease and possibly new therapeutic avenues.

\section{Acknowledgments}

We thank Tore Kempf for his advice in preparing two-dimensional gels, and Sabine Fiedler and Kerstin Kammerer for technical help with nanoLC ESI MS/MS. This work was supported financially as part of the Proteomics program, funded by the German Federal Ministry of Education and Research (BMBF), and the MolTools and MolDiagPaca projects of the European Commission.

The authors declare no competing interests.

\section{References}

1.Harry, J.L., M.R. Wilkins, B.R. Herbert, N.H.Packer, A.A. Gooley, and K.L. Williams.
2000. Proteomics: capacity versus utility. Electrophoresis 21:1071-1081.

2. Miklos, G.L. and R. Maleszka. 2001. Protein functions and biological contexts. Proteomics 1:169-178.

3. Hogeboom, G.H., W.C. Schneider, and G.E. Palade. 1948. Purification of diphosphopyridine nucleotide by counter-current distribution. J. Biol. Chem. 172:619-635.

4.Fleischer, B., S. Fleischer, and $H$. Ozawa. 1969. Isolation and characterization of golgi membranes from bovine liver. J. Cell Biol. 43:59-79.

5. Shaw, M.M. and B.M. Riederer. 2003. Sample preparation for two-dimensional gel electrophoresis. Proteomics 3:1408-1417.

6. Ramsby, M.L. and G.S. Makowski. 1999. Differential detergent fractionation of eukaryotic cells. Analysis by two-dimensional gel electrophoresis. Methods Mol. Biol. 112:5366.

7. Olsavsky, K.M., J.L. Page, M.C. Johnson, H. Zarbl, S.C. Strom, and C.J. Omiecinski. 2007. Gene expression profiling and differentiation assessment in primary human hepatocyte cultures, established hepatoma cell lines, and human liver tissues. Toxicol. Appl. Pharmacol. 222:42-56.

8. Yunis, A.A., G.K. Arimura, and D.J. Russin. 1977. Human pancreatic carcinoma (MIA PaCa-2) in continuous culture: sensitivity to asparaginase. Int. J. Cancer 19:128-135.

9. Ramana, K.V., D. Chandra, S. Srivastava, A. Bhatnagar, B.B. Aggarwal, and S.K. Srivastava. 2002. Aldose reductase mediates mitogenic signaling in vascular smooth muscle cells. J. Biol. Chem. 277:32063-32070.

10.Sinha, P., J. Poland, M. Schnolzer, and T. Rabilloud. 2001. A new silver staining apparatus and procedure for matrix-assisted laser desorption/ionization-time of flight analysis of proteins after two-dimensional electrophoresis. Proteomics 1:835-840.

11. Klimmeck, D., U. Mayer, N. Ungerer, U. Warnken, M. Schnölzer, S. Frings, and F. Möhrlen. 2008. Calcium-signaling networks in olfactory receptor neurons. Neuroscience 151:901-912.

12.Stahl, M., M. Retzlaff, M. Nassal, and J. Beck. 2007. Chaperone activation of the hepadnaviral reverse transcriptase for template RNA binding is established by the Hsp70 and stimulated by the Hsp90 system. Nucleic Acids Res. 35:6124-6136.

13. Leonard, W.J. 2001. Role of Jak kinases and STATs in cytokine signal transduction. Int. J. Hematol. 73:271-277.

14. David, V., F. Hochstenbach, S. Rajagopalan, and M.B. Brenner. 1993. Interaction with newly synthesized and retained proteins in the endoplasmic reticulum suggests a chaperone function for human integral membrane protein IP90 (calnexin). J. Biol. Chem. 268:9585-9592.

15.Guller, M., K. Toualbi-Abed, A. Legrand, L. Michel, A. Mauviel, D. Bernuau, and F. Daniel. 2008. C-Fos overexpression increases the proliferation of human hepatocytes by stabilizing nuclear Cyclin D1. World J. Gastroenterol. 14:6339-6346.

16. Foisner, R. 2003. Cell cycle dynamics of the nuclear envelope. ScientificWorldJournal 3:1-20.

17. Pittenger, J.T., J.F. Hess, M.S. Budamagunta, J.C. Voss, and P.G. Fitzgerald. 2008. Identification of phosphorylation-induced changes in vimentin intermediate filaments by site-directed spin labeling and electron paramagnetic resonance. Biochemistry 47:10863-10870.

18. Lehoux, E.A. and P.A. Fournier. 1999. Liquid $\mathrm{N}_{2}$ bath for the powdering of tissue with a mortar and pestle. Anal. Biochem. 269:213214.

19. Potter, V.R. and C.A. Elvehjem. 1936. A modified method for the study of tissue oxidations. J. Biol. Chem. 114:495-504.

20.Potter, V.R. 1946. The assay of animal tissues for respiratory enzymes IV: cell structure in relation to fatty acid oxidation. J. Biol. Chem. 163:437-446.

21. Brandhorst, H., D. Brandhorst, M.D. Brendel, B.J. Hering, and R.G. Bretzel. 1998. Assessment of intracellular insulin content during all steps of human islet isolation procedure. Cell Transplant. 7:489-495.

22.Swanson, C.J., B.J. Olack, D. Goodnight, L. Zhang, and T. Mohanakumar. 2001. Improved methods for the isolation and purification of porcine islets. Hum. Immunol. 62:739-749.

23. Bohman, S., A. Andersson, and A. King. 2006. No differences in efficacy between noncultured and cultured islets in reducing hyperglycemia in a nonvascularized islet graft model. Diabetes Technol. Ther. 8:536-545.

24.Mollenhauer, J., I. Roether, and H.F. Kern. 1987. Distribution of extracellular matrix proteins in pancreatic ductal adenocarcinoma and its influence on tumor cell proliferation in vitro. Pancreas 2:14-24.

25. Imamura, T., H. Iguchi, T. Manabe, G. Ohshio, T. Yoshimura, Z.H. Wang, H. Suwa, S. Ishigami, et al. 1995. Quantitative analysis of collagen and collagen subtypes I, III, and V in human pancreatic cancer, tumorassociated chronic pancreatitis, and alcoholic chronic pancreatitis. Pancreas 11:357-364.

26. Newton, S.S., A. Dow, R. Terwilliger, and R. Duman. 2002. A simplified method for combined immunohistochemistry and in-situ hybridization in fresh-frozen, cryocut mouse brain sections. Brain Res. Brain Res. Protoc. 9:214-219.

27. Boltze, C., R. Schneider-Stock, G. Aust, C. Mawrin, H. Dralle, A. Roessner, and C. Hoang-Vu. 2002. CD97, CD95 and Fas-L clearly discriminate between chronic pancreatitis and pancreatic ductal adenocarcinoma in perioperative evaluation of cryocut sections. Pathol. Int. 52:83-88.

28.Cooper, G.M. 2000. The Cell: A Molecular Approach, 2nd ed. Sinauer Associates Inc., Sunderland, MA.

29. Kwon, M., T.J. MacLeod, Y. Zhang, and D.M. Waisman. 2005. S100A10, annexin A2 and annexin a 2 heterotetramer as candidate plasminogen receptors. Front. Biosci. 10:300325.

Received 26 August 2008; accepted 29 December 2008.

Address correspondence to Anette Börner, Functional Genome Analysis, Deutsches Krebsforschungszentrum, Im Neuenheimer Feld 580, 69120 Heidelberg, Germany. email: a.boerner@dkfz.de 